\title{
Persona as a Tool to Involving Human in Agile Methods: Contributions from HCI and Marketing
}

\author{
Leydi Caballero' ${ }^{1}$, Ana M. Moreno ${ }^{2}$, and Ahmed Seffah ${ }^{3}$ \\ ${ }^{1}$ Instituto Tecnológico de Conkal, Km 16 Old Road Motul, Yucatán México \\ leydi.caballero@itconkal.edu.mx \\ ${ }^{2}$ Universidad Politécnica de Madrid, Campus Montegancedo 28660 Boadilla del Monte, Spain \\ ammoreno@fi.upm.es \\ ${ }^{3}$ University of Valenciennes, Le Mont Houy F59313 Valenciennes Cedex 9, France \\ ahmed.seffah@univ-valenciennes.fr
}

\begin{abstract}
Human centricity refers to the active involvement in the overall product lifecycle of different human actors including end-users, stakeholders and providers. Persona is one of the different tools that exist for human centricity. While marketing is the original domain in which persona was introduced, this technique has also been widely used in user-centered design (UCD) design. In these two perceptions, persona has demonstrated its potential as an efficient tool for grouping the users or customers and focusing on user or customer needs, goals and behavior. A segmentation technique is generally used with persona in order to group individual users according to their common features, identifying within these groups those that represent a pattern of human behavior. This paper investigates how persona has been used to improve the usability in the agile development domain, while studying which contributions from marketing and $\mathrm{HCI}$ have enriched persona in this agile context.
\end{abstract}

Keywords: Persona, Agile Usability, HCI methods in software engineering.

\section{Introduction}

The active involvement of customers, citizens and employees in the co-creation process of innovative solutions and products helps to understand human behaviors and factors that affect the quality of work, services and products. This active involvement can help to answer the designers and developers questions such as:

- What factors drive the use of the products or services? How do people actually use the services and in which context?

- What factors drive the adoption and acceptability of the product by a target community?

- What new services can we use or do we need to improve the communication line between the developers, brokers and users of a service?

- How does your target audience feel about themselves in the context of using your products or services? Do they look more satisfied, smart, or informed? 
The persona technique has been used successfully in marketing. In this context, this technique can give us a general scope of its advantages and disadvantages when the understanding of behavior of the final client is a crucial factor. Additionally, in recent years the concept of "persona" has been investigated as a powerful design tool focused on improving the design and usability of software development through the definition of user representations after learning and analyzing users' goals and behavior. The concept of "persona" in the HCI community was introduced by Alan Cooper in "The inmates are running the asylum" as part of his method Goal-Directed design (GDD) [1]. HCI focuses on improving software design from a usability perspective taking into account the user experience and skills. Looking for a new and better process to design Cooper [1] asserts that persona represents an efficient tool for communication and interaction.

On the other hand, agile methods constitute a software development approximation widely used nowadays [2, 3]. A core principle of the agile manifesto (www.agilemanifesto.org) emphasizes the continuous delivery of valuable software. This requires the correct identification of user needs and an adequate understanding of user priorities and goals [4]. Even when some voices in the agile community have claimed the adding values of HCI methods in explicitly developing for customer needs, in general, usability and user interaction factors, do not have yet the necessary relevance in the agile approximations. Among the supporters of the integration between HCI and agile, we find Ambler [5] who claims that good end product usability can be ensured only by systematic user centered design activities during the agile development iterations. Jokela and Abrahamsson [6] state that without explicit user experience practices added to agile methods, good software usability would be more or less a coincidence resulting from customer and/or developer intuition. Patton [7] pointed that before the first line of code can be written someone needs to decide how a specific user will interact with the software to achieve her goals. In sum, the use of HCI tools would help agile teams to identify properly and from the beginning of the development process the final users in order to develop for them. This would avoid waste of effort and time achieving the objectives defined by the principles of the Agile Manifesto. Persona is one of the HCI tools that have been used for this aim.

In what follows, we explore the characteristics and perceptions of persona in marketing, HCI and agile while identifying the contributions from the first two fields to the persona technique used in agile development.

\section{Persona in Consumer Research}

The essence of the marketing concept is to achieve the company's objectives by directing a coordinated marketing effort at identifying and satisfying consumer's wants and needs [8].

Segmentation was adopted to help marketers to better meet the needs of specific groups of consumers, dividing the total potential markets into smaller, homogeneous segments for which they could design specific products [9]. Solomon [10] describes the market segmentation as a process that identifies groups of customers who are similar to one another in one or more ways, and then devises marketing strategies. 
Engel et. al. [11] argue that each of us is a distinct market and the objective of segmentation is to identify groups within the broader market that are sufficiently similar in characteristics and responses to warrant separate treatment. Therefore, to better meet the customer's needs, the marketers must divide consumers in different groups (segmentation) according to common needs, attitudes or characteristics (consumer behavior).

In marketing research, the basic usage of persona arises from the concept of community of individuals introduced by Jenkinson [12]. He pointed that a group connotes a community of individuals, that is, individuals who have something in common. He specifies the difference between "segment" and "grouping". Segment implies dividing a population into groups using factors such as age, gender, interests, etc. Grouping means merging people to form a class of consumers who share common characteristics with tailored solutions.

In [13] Jenkinson proposed to implement an enriched segmentation model that the companies must communicate to staff and senior management converting the community segments into real living people using day-in-the-life archetype descriptions as Customer Prints or personas.

\section{Persona in $\mathrm{HCI}$}

Persona in HCI was presented as part of the effective mental tools considered in Cooper's method Goal-directed design. Cooper [1] describes persona as a powerful communication and interaction design tool. Although persona does not deal with real people they are represented along with their goals with significant rigor and precision synthesized directly from observations of real people [14]. Nielsen [15] pointed that personas are considered a method to communicate data about users and to aid in the perception of users. Instead of project participants having individual understandings they share a perception of the users that is built on field data and not on preconceived ideas.

Sharp et al. [16] describe the persona process related with Cooper's definition as the collection of attributes for a "typical user" called a user profile. The segments defined in marketing to learn and identify behaviors of the customers are applied, in a similar way, when the personas are defined in software design. Any product may have a number of different user profiles representing specific, individual human beings that the designers can focus on and design the product for [16]. These representations are analyzed and grouped by their similarities and this allows us to segmenting them and prioritizing, avoiding user's representations that eventually will not be representative users.

Like an actor represents a character in a movie or novel and through the history we understand their feelings, goals and behaviors, personas represent people who have their own stories that help the development team to understand the user. Quesenbery in [17] asserts that storytelling makes persona work; when we create a short anecdote to imagine how our persona might interact with our product we are creating a story that shows the persona in action and that helps to understand how to better design for him or her.

Despite the potential advantages of using persona, this technique has also been criticized mainly due to the fictitious or figurative descriptions generated to describe real people; using figurative models it can be difficult to know what is an accurate reflection of real user characteristics and what is mere concoction [17]. Another issue is the time consuming for persona during the development process [18]. 


\section{$4 \quad$ Persona in Agile Methods}

In order to explore how persona has been used in agile approximations we made a literature review. We searched in the following databases: IEEExplore Digital Library, Elsevier ScienceDirect, ACM digital library, Springer, Scopus. We queried these databases covering publications from mid-2001 until mid-2013 according to the following search criteria: (Usability OR Human-computer interaction OR User experience OR HCI) AND (Persona) AND (Agile OR Agile method OR Agile Development OR Agile Practice OR Agile Project OR SCRUM OR Extreme programming OR XP).

Table 1 shows a summary of the papers where we found relevant information about the use of persona in agile projects. In this table we show the source of the persona technique used by each author (mainly the HCI or the marketing version); the adaptation, if any, of persona to the agile domain; and the phase in the agile process where persona or its variants have been applied.

Regarding the phase in the agile development process where persona was applied, we identified two moments that we referred to as the Exploratory phase and the Refinement phase. During the Exploratory phase, personas are identified at the beginning of the project before working on any agile cycle and before any code is released. By this way a whole or partial perspective of the goals and speculations of the user is gathered to improve the results from the cycles or sprints derived and from the observations and feedbacks received. In the Refinement phase, that is, through the agile cycles, the personas defined in the Exploratory phase are evaluated using each cycle's feedback. This can help to determine if the personas must remain as were previously defined, be modified, eliminated or merged with others. In this phase, new personas can be also created.

Table 1. Persona in Agile projects

\begin{tabular}{|c|c|c|c|c|}
\hline Author/s & Ref. & $\begin{array}{l}\text { Source of } \\
\text { Persona }\end{array}$ & Concept & Agile Moment \\
\hline S. Chamberlain et. al. & [19] & HCI-Cooper & Persona & Exploratory phase \\
\hline J.Haikara 2007 & [20] & HCI-Cooper & Persona & $\begin{array}{l}\text { Exploratory phase } \\
\text { Refinement phase }\end{array}$ \\
\hline D.Sy 2007 & [21] & HCI-Cooper & Persona & Exploratory phase \\
\hline Z. Hussain et. al. & [22] & HCI-Cooper & Extreme Persona & $\begin{array}{l}\text { Exploratory phase } \\
\text { Refinement phase }\end{array}$ \\
\hline P.Wolkerstorfer et. al. & [23] & HCI-Cooper & Extreme Persona & $\begin{array}{l}\text { Exploratory phase } \\
\text { Refinement phase }\end{array}$ \\
\hline M.Najafi et. al. & [24] & HCI-Cooper & Persona & Exploratory phase \\
\hline M. Singh 2008 & [25] & $\begin{array}{c}\text { HCI-Cooper + } \\
\text { Marketing }\end{array}$ & User persona & Exploratory phase \\
\hline D.Broschinsky et.al. & [26] & $\begin{array}{c}\text { HCI-Cooper }+ \\
\text { contextual design }\end{array}$ & Persona & Exploratory phase \\
\hline L. Cho 2009 & [27] & HCI-Cooper & Persona & $\begin{array}{l}\text { Exploratory phase } \\
\text { Refinement phase }\end{array}$ \\
\hline J.Gonzalves et. al. & [28] & HCI-Cooper & Persona & Exploratory phase \\
\hline
\end{tabular}


In [19], Chamberlain and Sharp introduced "up-front design methods" where personas are defined based on significant user research carried out before any coding is done. Sy [21] also used persona at the start of the project, in particular in the cycle zero. The author claimed that this helps developing brief and vivid descriptions of target users and workflows. Similarly, Najafi et. al. [24] considered HCI methods in the sprint zero of a Scrum process. In this case, the user experience team specifically uses this sprint zero to understand the users, explore their context and identify their goals. They did these exploratory tasks using personas.

Singh [25] used Coopers' persona and describes a user persona as an archetypal user resulting from a combination of market research, ethnographic studies and anecdotal observations. The author proposes the U-Scrum model which incorporates a usability product owner from the beginning of the project. His role is to work with the Scrum product owner to achieve an agreement on the user experience. He works with the marketing team defining personas at the beginning of the process. In [26] personas were also brought before the development started to help developers know their users and become familiar with them. Personas descriptions are validated with customers and developers on-site. During the subsequent agile iterations, the personas are refined based on the revision meetings but preserving the essential of the original descriptions.

The software tool presented by Gonzalez et.al. [28]combines Scrum and Cooper's GDD methodology [14]. The purpose of the tool is to develop a system of low-fidelity prototyping and aims to help identifying and defining the personas as part of the cycle zero before the agile sprints.

Haikara [20] extended an interaction design process that exploits personas during the exploratory phase. In the first planning day, the primary persona is identified and subsequently in this phase persona expectations are refined. Subsequently persona definitions are used to keep in mind the persona definition in order to have a clear target audience that the development team should focus on.

Wolkerstorfer et. al. [22] and Hussain et. al. [23] extended the XP paradigm of small iterative steps and refactoring to persona resulting an "extreme persona". At the beginning of the process, the users are investigated through user studies to develop personas. At the end of each iteration the vision about the users is broadened. This helps in extending the personas for next cycle refactoring or if the knowledge reveals that current personas do not cover the new insights new personas will be developed.

In [27], the agile process uses Feature teams [29] self-organized to complete design, planning, and construction within the same sprint. Each sprint has explore and refinement phases. As the software evolved through the sprints, new functionalities are built into existing components. The initial set of persona is then updated or new personas are created.

\section{$5 \quad$ Concluding Remark}

We have seen how persona has been used in agile to help agile teams to get a good interaction design without breaking agile values and principles. Most agile authors that apply persona, use it according to the HCI philosophy for providing a more holistic model of the target users and their contexts. HCI advocates knowing and 
learning about the goals and behavior of the users at the beginning of the software development to identify the real end users and develop for them assuring by this way delivering useful software.

On the other hand, we have also seen how the marketing idea of persona as a vehicle for knowing the customer's behavior and for identifying the end customer that will use the product fits with the need to know the user's needs and goals in agile software development, to identify final users and develop for them. Particularly in agile the idea of segmentation has been applied with persona to identify the end users that will use the product delivered.

However, the original idea (from both HCI and marketing) regarding the creation of a complete up-front design that allows getting all the details from the final users of the system can be in contradiction with the agile philosophy. That's why the literature we surveyed showed that the traditional way of using persona has, in some cases, been adapted or "agilized"; allowing the partial application of the technique at the beginning of the agile project and its refinement and completion during the agile iterations. This agilized persona contributes to address the time restrictions that drive the agile development process.

The agile literature we have analyzed provides evidence that persona helps not only HCI designers to develop usable user interfaces but also agile developers and other stakeholders to elicit the client requirements and to engage client in the development lifecycle. We confirm the Cooper's [14] assertions that persona is powerful tool to:

- determine what a product should do and how it should behave,

- mediate the communicate line between stakeholders, developers and other designers,

- build consensus and commitment to the design from both the HCI and system/functionality perspectives,

- measure the design's effectiveness,

- contribute to other product-related efforts such as marketing and sales plans.

Persona is a flexible technique to be tailored for different development methods, projects and users.

\section{References}

1. Cooper, A.: Inmates Are Running the Asylum, The: Why High-Tech Products Drive Us Crazy and How to Restore the Sanity. Sams (1999)

2. Hussain, Z., Slany, W., Holzinger, A.: Investigating Agile User-Centered Design in Practice: A Grounded Theory Perspective. In: Holzinger, A., Miesenberger, K. (eds.) USAB 2009. LNCS, vol. 5889, pp. 279-289. Springer, Heidelberg (2009)

3. Dyba, T., Dingsoyr, T.: Empirical studies of agile software development: A systematic review. Inf. Softw. Technol. 50, 833-859 (2008)

4. Beck, K., Beedle, M., van Bennekum, A., Cockburn, A., Cunningham, W., Fowler, M.: Agile Manifesto, http: / / www .agilemanifesto.org 
5. Ambler, S.: Tailoring Usability into Agile Software Development Projects. In: Law, E.-C., Hvannberg, E., Cockton, G. (eds.) Maturing Usability SE - 4, pp. 75-95. Springer, London (2008)

6. Jokela, T., Abrahamsson, P.: Usability Assessment of an Extreme Programming Project: Close Co-operation with the Customer Does Not Equal to Good Usability. In: Bomarius, F., Iida, H. (eds.) PROFES 2004. LNCS, vol. 3009, pp. 393-407. Springer, Heidelberg (2004)

7. Patton, J.: Designing Requirements: Incorporating Usage-Centered Design into an Agile SW Development Process. In: Wells, D., Williams, L. (eds.) XP 2002. LNCS, vol. 2418, pp. 1-12. Springer, Heidelberg (2002)

8. Loudon, D.L., Della Bitta, A.J.: Consumer behavior: concepts and applications. McGrawHill, New York (1979)

9. Schiffman, L.G., Kanuk, L.L.: Consumer behavior. Prentice Hall, Upper Saddle River (1997)

10. Solomon, M.R.: Consumer behavior: buying, having, and being. Pearson Prentice Hall, Upper Saddle River (2006)

11. Engel, J.F., Blackwell, R.D., Miniard, P.W.: Consumer behavior. Dryden, Chicago (1990)

12. Jenkinson, A.: Beyond Segmentation. J. Targeting, Meas. Anal. Mark. 3, 60-72 (1994)

13. Jenkinson, A.: What happened to strategic segmentation? J. Direct, Data Digit. Mark. Pract. 11, 124 (2009)

14. Cooper, A., Reimann, R., Cronin, D.: About Face 3. The essentials of Interaction Design. Wiley Publishing (2007)

15. Nielsen, L.: Personas in Cross-Cultural Projects. In: Katre, D., Orngreen, R., Yammiyavar, P., Clemmensen, T. (eds.) Human Work Interaction Design: Usability in Social, Cultural and Organizational Contexts SE - 7, pp. 76-82. Springer, Heidelberg (2010)

16. Sharp, H., Rogers, Y., Preece, J.: Interaction Design beyond human-computer interaction. John Wiley \& Sons (2007)

17. Pruitt, J., Adlin, T.: The persona lifecycle: keeping people in mind throughout the product design. Morgan Kaufmann Publishers (2006)

18. McGinn, J. (J.), Kotamraju, N.: Data-driven persona development. In: Proceedings of the SIGCHI Conference on Human Factors in Computing Systems, p. 1521. ACM Press (2008)

19. Chamberlain, S., Sharp, H., Maiden, N.: Towards a framework for integrating agile development and user-centred design. In: Abrahamsson, P., Marchesi, M., Succi, G. (eds.) XP 2006. LNCS, vol. 4044, pp. 143-153. Springer, Heidelberg (2006)

20. Haikara, J.: Usability in Agile Software Development: Extending the Interaction Design Process with Personas Approach. In: Concas, G., Damiani, E., Scotto, M., Succi, G. (eds.) XP 2007. LNCS, vol. 4536, pp. 153-156. Springer, Heidelberg (2007)

21. Sy, D.: Adapting Usability Investigations for Agile User-centered Design. J. Usability Stud. 2, 112-132 (2007)

22. Hussain, Z., Lechner, M., Milchrahm, H., Shahzad, S., Slany, W., Umgeher, M., Wolkerstorfer, P.: Agile User-Centered Design Applied to a Mobile Multimedia Streaming Application. In: Holzinger, A. (ed.) USAB 2008. LNCS, vol. 5298, pp. 313-330. Springer, Heidelberg (2008)

23. Wolkerstorfer, P., Tscheligi, M., Sefelin, R., Milchrahm, H., Hussain, Z., Lechner, M., Shahzad, S.: Probing an agile usability process. In: Proceeding twenty-sixth Annu. CHI Conf. Ext. Abstr. Hum. factors Comput. Syst., CHI 2008, p. 2151 (2008)

24. Najafi, M., Toyoshiba, L.: Two Case Studies of User Experience Design and Agile Development. In: Agile 2008 Conference, pp. 531-536. IEEE Press (2008)

25. Singh, M.: U-SCRUM: An Agile Methodology for Promoting Usability. In: Agile 2008 Conference, pp. 555-560. IEEE (2008) 
26. Broschinsky, D., Baker, L.: Using Persona with XP at LANDesk Software, an Avocent Company. In: Agile 2008 Conference, pp. 543-548. IEEE (2008)

27. Cho, L.: Adopting an Agile Culture A User Experience Team's Journey. In: 2009 Agile Conference, pp. 416-421. IEEE (2009)

28. Gonçalves, J., Santos, C.: POLVO - Software for prototyping of low-fidelity interfaces in agile development. In: Jacko, J.A. (ed.) Human-Computer Interaction, Part I, HCII 2011. LNCS, vol. 6761, pp. 63-71. Springer, Heidelberg (2011)

29. Frank, A., Hartel, C.: Feature Teams Collaboratively Building Products from READY to DONE. In: Agile Conference, AGILE 2009, pp. 320-325 (2009) 